\title{
Conserved Growth on Vicinal Surfaces
}

\author{
Harald Kallabis \\ Center for Polymer Studies, Boston University, Boston, MA 02215, USA
}

May 30, 1998

\begin{abstract}
A crystal surface which is miscut with respect to a high symmetry plane exhibits steps with a characteristic distance. It is argued that the continuum description of growth on such a surface, when desorption can be neglected, is given by the anisotropic version of the conserved KPZ equation (T. Sun, H. Guo, and M. Grant, Phys. Rev. A 40, 6763 (1989)) with non-conserved noise. A one-loop dynamical renormalization group calculation yields the values of the dynamical exponent and the roughness exponent which are shown to be the same as in the isotropic case. The results presented here should apply in particular to growth under conditions which are typical for molecular beam epitaxy.
\end{abstract}

PACS numbers: 81.15-z, 81.10.Bk, 05.70.Ln 
The fabrication of novel electronic devices requires experimental conditions which are highly controllable. Molecular beam epitaxy (MBE) is a very valuable technology for this purpose. In this process particles are deposited under high vacuum conditions onto a crystal surface which usually has been cleaved prior to growth. The cleavage process may generate a surface which is miscut against a high-symmetry plane and which exhibits terraces with a characteristic width. The terrace size can be made very large, but a small miscut can never be avoided. Therefore the surface at the beginning of the growth proces has always to be regarded as vicinal.

Since desorption of adatoms can be neglected under experimental conditions which are typical for MBE, surface relaxation by adatom diffusion is volume conserving. In this paper I introduce the continuum description of such conserved growth on a vicinal surface. This allows for the analysis of the stochastic fluctations of the surface, which appear on large time and length scales during growth. The surface fluctuations, apart from being of interest in their own right, may be intimately related to the damping of oscillations observed during layer-by-layer growth, as has been shown recently [1, 2].

The surface fluctuations are expected to exhibit self-affine scaling [3]:

$$
w(t) \simeq a_{\perp}(\xi(t) / \tilde{\ell})^{\zeta} \quad \text { and } \quad \xi(t) \simeq \tilde{\ell}(t / \tilde{t})^{1 / z}
$$

if the surface is isotropic. Here $w$ is the root mean square variation of the film thickness (the surface width), $a_{\perp}$ the thickness of one atomic layer, and $\xi$ is the correlation length up to which the surface roughness has fully developed until time $t$. $\zeta$ is the roughness exponent and $z$ the dynamical exponent. The layer coherence length $\tilde{\ell}$ and the oscillation damping time $\tilde{t}$ [1] play the roles of natural cutoffs in the continuum description of the surface fluctuations at small length and time scales. To calculate the values of $z$ and $\zeta$, we derive the equation governing conserved growth on vicinal surfaces next.

On a coarse-grained scale the surface can be described at any given time $t$ by a singlevalued function $h\left(x_{\|}, x_{\perp}, t\right)$. The coordinate system is chosen such that the surface tilt is $m$ in $x_{\|}$-direction, while the steps are along the $x_{\perp}$-direction. It is then convenient to work 
in a tilted coordinate system, $h \rightarrow h-m x_{\|}$, so that $h$ represents the surface fluctuations around the average tilt. Since we consider conserved growth, the evolution equation for the surface has the form

$$
\partial_{t} h=-\nabla \cdot \mathbf{j}+\eta+\mathcal{F}
$$

with a surface diffusion current $\mathbf{j}$ and a noise term $\eta$ which models the disorder entering the mesoscopic description (2) due to the stochastic nature of the growth process. With the abbreviations $\partial_{\|} \equiv \partial / \partial_{\|}$and $\partial_{\perp} \equiv \partial / \partial_{\perp}$, the vector $\nabla$ reads $\left(\partial_{\|}, \partial_{\perp}\right)$. $\mathcal{F}$ is the average particle flux which is formally eliminated by changing to the comoving frame, $h \rightarrow h-\mathcal{F} t$. All lattice constants are set to unity for convenience.

The surface diffusion current has an equlibrium contribution and a nonequilibrium contribution, $\mathbf{j}=\mathbf{j}_{\text {eq }}+\mathbf{j}_{\text {neq }}$. $\mathbf{j}_{\text {eq }}$ is given by the tendency of the adatom current to even out gradients in the local equilibrium chemical potential $n_{\mathrm{eq}}$ of the surface, $\mathbf{j}_{\mathrm{eq}}=-\Gamma \nabla n_{\mathrm{eq}}$. $\Gamma$ is the adatom mobility, which for simplicity is assumed to be isotropic. $n_{\text {eq }}$ depends on $h$ like

$$
n_{\mathrm{eq}}=-\left(\frac{\kappa_{\|}}{\Gamma} \partial_{\|}^{2}+\frac{\kappa_{\perp}}{\Gamma} \partial_{\perp}^{2}\right) h
$$

$\kappa_{\|} / \Gamma$ and $\kappa_{\perp} / \Gamma$ are the anisotropic surface stiffnesses [ [ [6], which for small variation of the surface can be regarded as constant. Eq. (3) represents the Gibbs-Thomson effect.

The nonequilibrium contribution $\mathbf{j}_{\text {neq }}=-D \nabla n_{\text {neq }}$ to the surface current is driven by the nonequilibrium adatom density $n_{\text {neq }}$ [7]. $D$ is the (isotropic) diffusion constant. On a flat surface without steps, $n_{0}$ is of the order of $(F / D) \ell_{D}^{2}$, as derived in Ref. [8]. $\ell_{D}$ denotes the typical island distance on a flat surface [9]. We assume $\ell_{D}$ to be isotropic, which is the case if diffusion and lateral bonding of adatoms to islands is isotropic. When steps are present and the tilt is strong enough to suppress island nucleation on terraces, i.e. if $|m| \ell_{D} \gtrsim 1, n_{0}$ depends on the local surface tilt: $n_{0} \propto(\mathcal{F} / D) /|m|^{2}$. A convenient Ansatz for interpolation between those two regimes is 10

$$
n_{\text {neq }}(\nabla h)=\frac{n_{0}}{1+\ell_{D}^{2}\left[\left(m+\partial_{\|} h\right)^{2}+\left(\partial_{\perp} h\right)^{2}\right]}
$$


in our coordinate system. $n_{\text {neq }}$ can be expanded for small deviations from the global tilt to give

$$
n_{\text {neq }}(\nabla h) \simeq n_{\text {neq }}(0)-\frac{\mu_{\|}}{D} \partial_{\|} h-\frac{\lambda_{\|}}{D}\left(\partial_{\|} h\right)^{2}-\frac{\lambda_{\perp}}{D}\left(\partial_{\perp} h\right)^{2}
$$

with the quantities $n_{\text {neq }}(0), \mu_{\|}$, and $\lambda_{\perp}$ being positive functions of $|m|, n_{0}$, and $\ell_{D}$. For small tilts, $|m| \ell_{D} \lesssim 1, \lambda_{\|}$is positive, while for large tilts, $|m| \ell_{D} \gtrsim 1$, it is negative. These two cases distingush between nucleation-dominated growth and step-flow growth, respectively. The different signs of the nonlinearities are known to have dramatic consequences for the surface fluctuations in the case of nonconserved growth on vicinal surfaces, which is described by the anisotropic KPZ equation [11]. One aim of the present study is to see if a similar scenario can be found in the conserved case.

The noise $\eta$ has three contributions in MBE growth [9]. Those are shot noise, diffusion noise, and nucleation noise. Shot noise arises due to statistical fluctuations in the atom beam which can be assumed to be isotropic. Diffusion noise has its origin in the stochastic motion of adatoms. Since we assume diffusion to be isotropic, this contribution is isotropic as well. Finally, nucleation noise describes the random distribution of island nucleation locations. Because diffusion noise and shot noise together generate nucleation noise, the latter is also isotropic. In Ref. [12] it has been shown that nucleation noise is long-range correlated in time as long as the surface grows layerwise. The continuum approach we pursue here is applicable for times larger than the oscillation damping time, which marks the transition from layer-by-layer growth to rough growth. After this time, the temporal correlations have ceased. Therefore we can assume nucleation noise to be short-range correlated in time for the present purpose. Note also that despite their relation on the microscopic level, there are no correlations between the different kinds of noise. With these remarks, the noise correlator reads 9,13

$$
\langle\eta(x, t) \eta(y, s)\rangle=\left[\mathcal{F}-\mathcal{D} \nabla^{2}+\mathcal{N}\left(\nabla^{2}\right)^{2}\right] \delta^{2}(x-y) \delta(t-s)
$$

$\mathcal{F}, \mathcal{D}$, and $\mathcal{N}$ denote the strengths of the shot noise, the diffusion noise, and the nucleation noise, respectively. The average value $\langle\eta\rangle$ vanishes. 
In summary, we arrive at the anisotropic conserved KPZ (ACKPZ) equation

$$
\partial_{t} h=-\nabla^{2}\left[\left(\kappa_{\|} \partial_{\|}^{2}+\kappa_{\perp} \partial_{\perp}^{2}\right) h+\mu \partial_{\|} h+\lambda_{\|}\left(\partial_{\|} h\right)^{2}+\lambda_{\perp}\left(\partial_{\perp} h\right)^{2}\right]+\eta
$$

where the noise correlator is given by Eq. (6). Note that the linear term proportional to $\mu$ cannot be transformed away as in the nonconserved case [11. This equation with $\mu=0$ has first been studied in Ref. [14]. However, as the following analysis shows, the conclusion presented there has to be corrected. We study the surface fluctuations predicted by Eq. (7) next.

In the linear case $\lambda_{\|}=\lambda_{\perp}=0$, Eq. (7) can be solved directly. One result is that the term $\propto \mu$ does not influence the surface fluctuations. Setting $\mu=0$, we may get the values of the exponents by rescaling

$$
x_{\perp} \rightarrow b x_{\perp}, \quad x_{\|} \rightarrow b^{\chi} x_{\|} \quad t \rightarrow b^{z} t, \quad h \rightarrow b^{\zeta} h,
$$

where $b$ is an arbitrary scaling factor [15]. The anisotropy exponent $\chi$ [11, 16] has to be introduced here to account for the fact that in contrast to Eq. (11), which is isotropic, there may be different characteristic lengths $\xi_{\|}$and $\xi_{\perp}$ governing the morphology of the surface. $\chi$ is defined by the relation $\xi_{\|} \propto \xi_{\perp}^{\chi}$. By writing $b=\exp (d \ell)$ with infintesimal $d \ell$, we get

$$
\begin{aligned}
& \frac{d \kappa_{\|}}{d \ell}=\kappa_{\|}(z-4 \chi) \\
& \frac{d r_{\kappa}}{d \ell}=4 r_{\kappa}(1-\chi) \\
& \frac{d \mathcal{F}}{d \ell}=\mathcal{F}(z-2 \zeta-2) \\
& \frac{d \mathcal{D}}{d \ell}=\mathcal{D}(z-2 \zeta-4) \\
& \frac{d \mathcal{N}}{d \ell}=\mathcal{N}(z-2 \zeta-6)
\end{aligned}
$$

for the change of the parameters in the continuum equation upon rescaling, where $r_{\kappa} \equiv$ $\kappa_{\|} / \kappa_{\perp}$. From Eqns. (11), (12), and (13) we see that shot noise is the most relevant type of noise, since it grows the fastest upon an increase of scale for any values of $z$ and $\zeta$. For this reason, we have to use Eq. (11) in the determination of the exponents. Requiring scale 
invariance of the surface amounts to setting the left-hand sides of the above equations to zero. Using Eqns. (9), (10), and (11), we get the exponents

$$
z=4, \quad \zeta=1, \quad \chi=1
$$

This means that a growing surface described by the linear version of Eq. (7) can only be scale-invariant if the two spatial coordinates are rescaled with the same scaling factor $b$. Then, the surface fluctuations are governed by the isotropic version of Eq. (7) with $\lambda_{\|}=0$ and $\lambda_{\perp}=0$.

The full (nonlinear) Eq. (7) is dealt with along the lines described in Refs. [15,17]: Wavenumbers $b \pi / a \leq\left|k_{\perp}\right| \leq \pi / a$ and $b^{\chi} \pi / a \leq\left|k_{\|}\right| \leq \pi / a$, where $a$ is the lattice constant parallel to the surface, are integrated out ( $a$ is set to one for convenience). This is done in a one-loop approximation published in detail elsewhere 18]. The resulting renormalized parameters are then rescaled according to Eq. (8). It turns out that $\mathcal{F}, \lambda_{\|}$, and $\lambda_{\perp}$ are not renormalized. The corresponding flow equations are

$$
\begin{aligned}
& \frac{d \lambda_{\|}}{d \ell}=\lambda_{\|}(z-4 \chi+\zeta) \\
& \frac{d r_{\lambda}}{d \ell}=r_{\lambda}(1-\chi)
\end{aligned}
$$

and Eq. (11), where $r_{\lambda} \equiv \lambda_{\|} / \lambda_{\perp}$. Those three equations already fix the exponents to be

$$
z=\frac{10}{3} \quad \text { and } \quad \zeta=\frac{2}{3}
$$

if the system is scale invariant and if $\lambda_{\|}, \lambda_{\perp} \neq 0$. These are the values for the isotropic conserved KPZ equation in $d=2[13,19$ in one-loop order. (In two-loop order they are slightly modified [20].)

The one-loop corrections to the parameters do not depend on $\mu$ [18]. Thus the corresponding term does not play a role in the determination of the surface fluctuations — as in the linear case. (Interestingly this does not hold for a related deterministic nonlinear equation which exhibits deterministic chaos [21].) For this reason, we set $\mu=0$ in the following. 
A remark on the noise renormalization is in order. The nucleation noise is renormalized and the corresponding flow equation reads

$$
\frac{d \mathcal{N}}{d \ell}=\mathcal{N}[z-2 \zeta-6]+g_{\perp} f\left(\kappa_{\|}, \kappa_{\perp}, \lambda_{\|}, \lambda_{\perp}, \mathcal{F}, \mathcal{D}, \mathcal{N}\right)
$$

with $g_{\perp} \equiv(2 \pi)^{-2} \mathcal{F} \lambda_{\perp}^{2} / \kappa_{\perp}^{3} . \quad f$ is non-negative for non-negative $\mathcal{F}$ or $\mathcal{D}$ [18. This means that, even if nucleation noise were absent $(\mathcal{N}=0)$ initially, this type of noise would automatically be generated by deposition and diffusion noise - which is immediately clear in the microscopic picture. As mentioned above, however, we utilize Eq. (11) for the determination of the exponents.

$\kappa_{\|}$and $\kappa_{\perp}$ also are renormalized. This can be discussed most conveniently by considering the flow equations for $r_{\kappa}$ and $g_{\perp}$ :

$$
\begin{aligned}
\frac{d r_{\kappa}}{d \ell}= & \frac{g_{\perp} \pi}{4 r_{\kappa}^{3 / 2}\left(r_{\kappa}-1\right)^{2}}\left[9\left(r_{\lambda}^{2}-r_{\kappa}^{4}\right)+r_{\kappa}^{3}\left(26-8 r_{\lambda}\right)\right. \\
& \left.+r_{\kappa} r_{\lambda}\left(8-26 r_{\lambda}\right)+16\left(r_{\lambda}-1\right)\left(r_{\kappa}^{5 / 2}+r_{\kappa}^{3 / 2} r_{\lambda}\right)+r_{\kappa}^{2}\left(r_{\lambda}^{2}-1\right)\right] \\
\frac{d g_{\perp}}{d \ell}= & g_{\perp}\left[2-\frac{3}{4} g_{\perp} \pi \frac{9 r_{\kappa}^{3}+r_{\kappa}^{2}\left(7 r_{\lambda}-26\right)-16 r_{\kappa}^{3 / 2}\left(r_{\lambda}-1\right)+r_{\kappa}\left(10 r_{\lambda}+1\right)-r_{\lambda}}{r_{\kappa}^{3 / 2}\left(r_{\kappa}-1\right)^{2}}\right] .
\end{aligned}
$$

Note that in the limit of $r_{\lambda}, r_{\kappa} \rightarrow 1$, these flow equations reduce to the isotropic case as obtained in Ref. [13]. Now the only important feature of these equations is if the fixed point $g_{\perp}^{*}=0$ is stable. If so, the nonlinearities would vanish on large scales and the growth exponents would take the values (14) given by the linear equation. This scenario is found for the anisotropic KPZ equation [1]. Here we want to find out if the ACKPZ equation shows the same behaviour.

For fixed $r_{\kappa}$, it is clear from Eq. (20) that $g_{\perp}^{*}=0$ is unstable, since $d g_{\perp} / d \ell=2 g_{\perp}$ for small $g_{\perp}$. Since equations (19) and (20) are coupled, however, $g_{\perp}^{*}=0$ could in principle be reached for $r_{\lambda}<0$ if $r_{\kappa}$ vanishes together with $g_{\perp}$ in a suitable way, see Eq. (20). To see if this is possible, we consider the limit $r_{\kappa} \rightarrow 0$ of (19) and (20),

$$
\frac{d r_{\kappa}}{d \ell}=\frac{9 \pi g_{\perp} r_{\lambda}^{2}}{4 r_{\kappa}^{3 / 2}}
$$




$$
\frac{d g_{\perp}}{d \ell}=g_{\perp}\left[2+\frac{3 \pi g_{\perp} r_{\lambda}}{4 r_{\kappa}^{3 / 2}}\right]
$$

To simplify the notation we set $r_{\lambda}=-8 /(3 \pi)$. For an initial condition $g_{\perp}>r_{\kappa}^{3 / 2}$, the coupling constant $g_{\perp}$ will decrease initially, see Eq. (22). For this decrease to continue, $g_{\perp} / r_{\kappa}^{3 / 2}>1$ has to hold. Eq. (21) then shows that $r_{\kappa}$, and consequently $g_{\perp}$ will increase again. Consequently, the fixed point $g_{\perp}^{*}=0$ is always unstable. Thus we can summarize that a change of universality class from the nonlinear to the linear equation is not possible.

In conclusion I have argued that the ACKPZ equation describes the fluctuations of a vicincal surface growing under MBE conditions. The dynamical and the roughness exponents are those of the isotropic conserved KPZ equation. In particular, the change from the nonlinear to the linear universality class, as observed for the anisotropic KPZ equation [11], is not found here. Corroboration of these findings in experiments or computer simulations are left for future research.

Acknowledgements. I thank Lothar Brendel and Martin Rost for very useful interactions and a critical proofreading of the manuscript. I am grateful to Joakim Hove for making available his diploma thesis. I acknowledge support by the German Academic Exchange Service within the Hochschulsonderprogramm III. 


\section{REFERENCES}

[1] H. Kallabis, L. Brendel, J. Krug and D. E. Wolf, Int. J. Mod. Phys. B 11, 3621 (1997).

[2] M. Rost and J. Krug, J. Phys. I (France) 71627 (1997).

[3] F. Family and T. Vicsek (eds.): Dynamics of Fractal Surfaces (World Scientific, Singapore 1991).

[4] M. Rost, P. Šmilauer, J. Krug, Surf. Sci. 369, 393 (1996).

[5] J. Krug, H. T. Dobbs, and S. Majaniemi, Z. Phys. B 97, 281 (1995).

[6] P. Noziéres, in: C. Godrèche (ed.), Solids far from equilibrium, Cambridge University Press (1991).

[7] J. Villain, J. Phys. (France) I 1, 19 (1991).

[8] J. Villain, A. Pimpinelli and D. Wolf, Comments Cond. Mat. Phys. 16, 1 (1992).

[9] D. E. Wolf, in: A. McKane et al. (eds.), Scale Invariance, Interfaces and NonEquilibrium Dynamics, Plenum Press, New York (1995).

[10] P. Politi and J. Villain, Phys. Rev. B 54, 5114 (1996).

[11] D. E. Wolf, Phys. Rev. Lett. 67, 1783 (1991).

[12] E. Somfai, J. Kertész and D. E. Wolf, J. Phys. I France 6, 393 (1996).

[13] L.-H. Tang and T. Nattermann, Phys. Rev. Lett. 66, 2899 (1991).

[14] J. Hove, diploma thesis, Trondheim, Norway (1996).

[15] E. Medina, T. Hwa, M. Kardar, and Y.-C. Zhang, Phys. Rev. A 39, 3053 (1989).

[16] T. Hwa and M. Kardar, Phys. Rev. Lett. 62, 1813 (1989).

[17] D. Forster, D. R. Nelson and M. J. Stephen, Phys. Rev. A 16, 732 (1977).

[18] H. Kallabis, Ph. D. dissertation, Universität Duisburg, and Juel-Bericht 3484, 
Forschungszentrum Jülich (1997). Some of the integrals in this calculation have been computed with Maple V Release 4, Waterloo Maple Inc. (1996).

[19] T. Sun, H. Guo and M. Grant, Phys. Rev.A 40, 6763 (1989).

[20] H. K. Janssen, Phys. Rev. Lett. 78, 1082 (1997).

[21] M. Sato and M. Uwaha, Europhys. Lett. 32, 639 (1995). 